\title{
On the completeness of root functions of elliptic boundary problems in a domain with conical points on the boundary
}

\author{
Yu. V. Egorov, V. A. Kondratiev, and B.-W. Schulze*
}

\section{Introduction.}

In this paper we prove the completeness of the system of eigen and associated functions (i.e., root functions) of an elliptic boundary value problem in a domain whose boundary is a smooth surface everywhere except for a finite number of points such that each point has a neighborhood where the boundary is a conical surface.

The problem of completeness of the system of eigen and associated functions of boundary value problems for elliptic operators in domains with smooth boundaries has been studied by numerous authors. F.B. Browder [1]-[3] obtained the theorem for the Dirichlet problem for elliptic operators of any order with a real principal part.

Earlier M.V. Keldysh [4] proved the general theorem on the completeness of the system of eigen and associated functions of differential not self-adjoint operators and obtained as its corollary the theorem on the completeness for elliptic operators of second order with Dirichlet boundary conditions.

For the Dirichlet problem for strongly elliptic differential operators of order $2 m$ the completeness of the system of eigen and associated functions in $L_{2}(\Omega)$, where $\Omega$ is an arbitrary bounded domain, was proved by M.S. Agranovich [5]. He studied also the problem with Neumann conditions, for the case of a Lipschitz boundary $\partial \Omega$. The problem for elliptic systems of second order was studied by N.M. Krukovsky [6].

All these authors referred to the methods of M.V. Keldysh [4]. We also use them here, together with the approach of T. Carleman as in [7].

S. Agmon [8] and M. Schechter [9] proved that the system of root functions of an elliptic boundary problem is complete in a bounded domain $\Omega$ with a smooth

\footnotetext{
*Corresponding author: e-mail: schulze@math.uni-potsdam.de, Phone: +49 3319771269 , Fax: +49 3319771440
} 
boundary if the Lopatinsky conditions are fulfilled. R. Denk, M.S. Agranovich, H. Faierman [11] improved Agmon's theorem under weaker regularity conditions for the boundary.

In [21] the authors have proved the theorem on completeness of the root functions for an elliptic operator on a closed manifold with conic points. (In this paper the term "paracompact" is to be replaced by "compact"; the authors are grateful to M.S. Agranovich for that remark.)

\section{Definitions}

Let $\Omega$ be a bounded domain in $\mathbf{R}^{n}$ and $\partial \Omega$ its boundary, $\bar{\Omega}$ the closure of $\Omega$. We shall use the standard notation $: x=\left(x_{1}, \ldots x_{n}\right), D^{\alpha}=\frac{\partial^{|\alpha|}}{\partial x_{1}^{\alpha} \ldots \partial x_{n}^{\alpha_{n}}}$ for a multi-index $\alpha=\left(\alpha_{1}, \ldots \alpha_{n}\right)$, where $|\alpha|=\alpha_{1}+\cdots+\alpha_{n}$.

Assume that $\partial \Omega$ is a surface of the class $C^{2 m}$ everywhere except for the point $x=0$ (denoted by $O$ ) and that it coincides in a neighborhood of the point $O$ with a conical domain $K=\left\{x: \frac{x}{|x|} \in K^{\prime}\right\}$, where $K^{\prime}$ is a domain on the unit sphere having a boundary of the class $C^{2 m}$.

Consider a differential operator in $\Omega$ :

$$
L(x, D)=\sum_{|\alpha| \leq 2 m} a_{\alpha}(x) D^{\alpha},
$$

where $a_{\alpha}(x)$ are bounded measurable functions in $\bar{\Omega}$, and for $|\alpha|=2 m$ they are continuous in $\bar{\Omega} \backslash O$.

The coefficients $a_{\alpha}(x)$ for $|\alpha|=2 m$ are assumed to have the form

$$
a_{\alpha}(x)=a_{\alpha 0}\left(\frac{x}{|x|}\right)+a_{\alpha 1}(x),
$$

in a neighborhood of the point $O$, where $\lim _{x \rightarrow 0} a_{\alpha 1}(x)=0$.

Set

$$
B_{j}(x, D)=\sum_{|\alpha| \leq m_{j}} b_{\alpha j}(x) D^{\alpha}, \quad j=1, \ldots m, \quad m_{j}<2 m,
$$

where $b_{\alpha j}(x)$ are functions of the class $C^{2 m-j}$ in $\bar{\Omega} \backslash O$, and for $|\alpha|=m_{j}$

$$
b_{\alpha j}(x)=b_{\alpha j 0}\left(\frac{x}{|x|}\right)+b_{\alpha j 1}(x) \text {, }
$$

where $\lim _{x \rightarrow 0} b_{\alpha j 1}(x)=0$.

Let $L^{\prime}=\sum_{|\alpha|=2 m} a_{\alpha}(x) D^{\alpha}$.

We will suppose everywhere below that the operator $L$ is elliptic, i.e. $L^{\prime}(x, \xi) \neq$ $0, \quad \xi \in \mathbf{R}^{n} \backslash 0, \quad x \in \bar{\Omega} \backslash O$. If $n=2$, we assume that the condition of regular ellipticity holds. It means that for any pair of linearly independent vectors $\xi, \eta$ and 
$x \in \Omega$ the polynomial $A^{\prime}(x, \xi+t \eta)$ has exactly $m$ roots $t$ with positive imaginary part. It is known (see [10]) that this condition is always satisfied for $n>2$. It holds also if the coefficients of the operator $L^{\prime}$ are real.

Suppose that the Lopatinsky condition (see [10]) is fulfilled outside the point $x=0$. The operators

$$
L_{0}=\sum_{|\alpha|=2 m} a_{\alpha 0}(\omega) D^{\alpha}, \quad B_{j 0}=\sum_{|\alpha|=m_{j}} b_{\alpha j 0}(\omega) D^{\alpha},
$$

where $(\rho, \omega)$ is the spherical coordinate system with its center in $O$, satisfy the Lopatinsky condition on $\partial K^{\prime} \backslash O$.

We shall consider complex-valued functions defined in $\Omega$. For $u \in C^{k}(\bar{\Omega})$ we introduce the norm

$$
\|u\|_{W_{2}^{k}(\Omega)}=\left(\int_{\Omega} \sum_{|\alpha| \leq k}\left|D^{\alpha} u\right|^{2} d x\right)^{1 / 2} .
$$

The Banach space $W_{2}^{k}(\Omega)$ is defined to be the completion of the space $C^{k}(\bar{\Omega})$ with respect to this norm.

The space $W_{\gamma}^{k}(\Omega)$ consists of the functions $u$ such that

$$
\|u\|_{W_{\gamma}^{k}(\Omega)}^{2} \equiv \sum_{|\alpha| \leq k} \int_{\Omega}|x|^{\gamma-2|\alpha|+2 k}\left|D^{\alpha} u\right|^{2} d x<\infty .
$$

Let us consider the boundary problem

$$
\begin{array}{ll}
L(x, D) u=f(x), & x \in \Omega ; \\
B_{j}(x, D) u=0, & x \in \partial \Omega \backslash O, \quad j=1, \ldots m .
\end{array}
$$

The following operator pencil is very important for the study of the boundary problems in domains with a conical point on the boundary :

$$
r^{-\lambda} L_{0}\left(r^{\lambda} \Phi(\omega)\right), \quad \omega \in K^{\prime} ; \quad r^{-\lambda} B_{j 0}\left(r^{\lambda} \Phi(\omega)\right), \quad \omega \in \partial K^{\prime},
$$

where

$$
L_{0}(\omega D)=\sum_{|\alpha|=m} a_{\alpha 0}(\omega) D^{\alpha}, \quad B_{j 0}(\omega D)=\sum_{|a|=m_{j}} b_{\alpha j 0}(\omega) D^{\alpha} .
$$

It is well known that the spectrum of the problem

$$
\begin{aligned}
r^{-2 m \lambda} L_{0}\left(\omega, r^{\lambda} D\right) u=0, & \omega \in K^{\prime} \\
r^{-m_{j} \lambda} B_{j 0}\left(\omega, r^{\lambda} D\right) u & =0, \quad \omega \in \partial K^{\prime},
\end{aligned}
$$

is discrete.

The following theorem is proved in [14] (see also [15]). 
Theorem 1. If there are no points of the spectrum of the problem (2) on the line $\operatorname{Re} \lambda=\frac{-\gamma+4 m-n}{2}$, then

$$
\|u\|_{W_{\gamma}^{2 m}(\Omega)} \leq C\left(\|L u\|_{W_{\gamma}^{0}(\Omega)}+\|u\|_{L_{2}(\Omega)}\right)
$$

for all functions $u(x) \in W_{\gamma}^{2 m}(\Omega)$ such that $\quad B_{j} u=0, \quad j=1, \ldots m, \quad x \in$ $\partial \Omega \backslash O$.

\section{Rays of minimal growth}

Let us denote $\mathcal{L}$ the linear unbounded operator $L_{2}(\Omega) \rightarrow L_{2}(\Omega)$, defined in $D_{\mathcal{L}}=$ $\left\{u: u \in W_{\gamma}^{2 m}(\Omega), B_{j} u=0, x \in \partial \Omega \backslash O\right\}$, which transforms each element $u \in D_{\mathcal{L}}$ to $L u$.

Theorem 1 implies that $\mathcal{L}$ is a closed linear operator $L_{2}(\Omega) \rightarrow L_{2}(\Omega)$, and the dimensions of its kernel and cokernel are finite. If the spectrum of $\mathcal{L}$ is not the whole complex plane then it is discrete.

Definition. A ray $\arg \lambda=\theta$ of the complex plane $\lambda$ is a ray of minimal growth for the resolvent $R(\lambda)=(\mathcal{L}-\lambda E)^{-1}: L_{2}(\Omega) \rightarrow L_{2}(\Omega)$ of the operator $\mathcal{L}$, if the resolvent does exist for all $\lambda$ on this ray with sufficiently large absolute value, and for all such $\lambda$ we have

$$
\|R(\lambda)\|_{L_{2}(\Omega) \rightarrow L_{2}(\Omega)} \leq C|\lambda|^{-\delta}, \quad \delta>0
$$

where $C=$ const $>0, \quad \delta=$ const $>0$.

Note that this definition is slightly different from the usual one when one assumes that $\delta=1$.

We will indicate now some conditions when a ray $\arg \lambda=\theta$ is a ray of minimal growth for the operator $\mathcal{L}$.

Theorem 2. The spectrum of the operator $\mathcal{L}$ is discrete and the ray $\arg \lambda=\theta$ is a ray of minimal growth for $R(\lambda, \mathcal{L})$ if the following conditions are fulfilled:

1) $(-1)^{m} \frac{L_{0}(x, \xi)}{\left|L_{0}(x, \xi)\right|} \neq e^{i \theta}, \quad \xi \neq 0, \quad \xi \in \mathbf{R}^{n}, \quad x \in \Omega$.

2) At each point $x \in \partial \Omega \backslash O$ denote $\nu$ the normal vector to $\partial \Omega$ and let $\xi \neq 0$ be a real vector, orthogonal to $\nu$. Let $t_{1}^{+}(\xi, \lambda), k=1, \ldots m$, be the roots with positive imaginary parts of the polynomial in $t$

$$
(-1)^{m} L_{0}(x, \xi+t \nu)-\lambda
$$

where $\lambda$ is a complex number such that $\arg \lambda=\theta$. Then the polynomials $B_{j \nu}(x, \xi+$ $t \nu), j=1, \ldots m$, are linearly independent modulo the polynomial $\Pi_{k=1}^{m}\left(t-t_{k}^{+}(\xi, \lambda)\right)$. 3) The boundary value problem in the infinite cone $K$

$$
L_{0}\left(\omega, D_{x}\right) u-\lambda u \equiv \sum_{|\alpha|=2 m} a_{\alpha}(\omega) D^{\alpha} u-e^{i \theta} u=f \in W_{\gamma}^{0}(K)
$$




$$
B_{j 0}\left(\omega, D_{x}\right) u=\sum_{|\alpha|=m_{i}} a_{\alpha_{i} 0}(\omega) D^{\alpha} u=0, \quad \text { on } \partial K
$$

has a unique solution from the class $W_{\gamma}^{2 m}(K) \cap W_{\gamma}^{0}(K)$ for some $\gamma, 0 \leq \gamma<2 m$, and

$$
\|u\|_{W_{\gamma}^{2 m}(K)}+\|u\|_{W_{\gamma}^{0}(K)} \leq C\|f\|_{W_{\gamma}^{0}(K)} .
$$

The condition 3 ) is difficult to check. It appears systematically in the study of the problem about the solvability of boundary problems in domains with singularities of the type of edge on its boundary. (See, for example, [18], [19]). It is possible to show that the problem (3) is Fredholm, i.e., its kernel and cokernel have finite dimensions. Condition 3) says that these dimensions are equal to zero.

This condition is equivalent to the following one :

3) Consider the boundary problem

$$
\begin{gathered}
L_{0} u-(-1)^{m} e^{i \theta} \frac{\partial^{2 m}}{\partial x_{n+1}^{2 m}} u=f \quad \text { in } K \times \mathbf{R}^{1}, \\
B_{j 0} u=0, \quad \text { on } \partial K \times \mathbf{R}^{1}, j=1, \ldots, m .
\end{gathered}
$$

Suppose that for any $f(x)$ such that

$$
\int_{K \times \mathbf{R}} r^{\gamma}|f|^{2} d x d x_{n+1}<\infty, \quad r^{2}=\sum_{i=1}^{n} x_{i}^{2}
$$

there exists a unique solution such that

$$
\int_{K \times \mathbf{R}^{1}} r^{\gamma-4 m}|u|^{2} d x d x_{n+1}<\infty .
$$

Conditions 1), 2) were introduced by Agmon in [8], where the completeness of the system of eigen and associated (root) vectors was proved for an elliptic boundary problem in a smooth domain. These conditions appear in the study of an elliptic boundary problem with a parameter. In the condition 2) the existence of $m$ roots with positive imaginary parts follows from the ellipticity of the operator $L$, which implies also that their number is less or equal to $m$. Condition 3 ) is connected with the presence of a conical point on the boundary. It is stated here not effectively as in the articles $[18],[19],[20]$, where the boundary value problem was considered in the domain with an edge on its boundary.

\section{Proof of Theorem 2.}

We have to show that the estimate

$$
\|u\|_{L_{2}(\Omega)} \leq \frac{C}{|\lambda|^{\delta}}\|(\mathcal{L}-\lambda E) u\|_{L_{2}(\Omega)}
$$


holds for any functions $u \in D_{\mathcal{L}}$ and all $\lambda$ with sufficiently large absolute value on the ray $\arg \lambda=\theta, \delta>0$.

Consider the operator

$$
L^{0} \equiv L-(-1)^{m} e^{i \theta} \frac{\partial^{2 m}}{\partial x_{n+1}^{2 m}} .
$$

The operator $L^{0}$ is elliptic of order $2 m$ in the closure of the cylindrical domain $\Omega_{0}=\left\{\left(x, x_{n+1}\right): x \in \Omega,-\infty<x_{n+1}<+\infty\right\}$. One can check that condition 2$)$ is equivalent to the condition that the operator $L^{0}$ and the system of boundary operators $B_{j}$ satisfy the Lopatinsky condition at each point of $(\partial \Omega \backslash O) \times(-\infty,+\infty)$.

Let $u\left(x, x_{n+1}\right) \in C^{2 m}\left(\bar{\Omega}_{0}\right)$ be such that $u \equiv 0$ for $\left|x_{n+1}\right| \geq 1$ and

$$
B_{j} u=0, \quad j=1, \ldots m \quad \text { on } \quad(\partial \Omega \backslash O) \times(-\infty,+\infty) .
$$

Then the following estimate

$$
\begin{aligned}
& \sum_{|\alpha| \leq 2 m} \int_{\Omega_{0}} r^{-4 m+2|\alpha|+\gamma}\left|D^{\alpha} u\right|^{2} d x d x_{n+1}+\int_{\Omega_{0}} r^{\gamma}\left|\frac{\partial^{2 m} u}{\partial x_{n+1}^{2 m}}\right|^{2} d x d x_{n+1} \\
& \leq c \int_{\Omega_{0}}\left|L^{0} u\right|^{2} r^{\gamma} d x d x_{n+1}+c \int_{\Omega_{0}}|u|^{2} d x d x_{n+1}
\end{aligned}
$$

is true with a constant $c$ independent of $u$. Estimate (5) can be proved with the help of a partition of the unity and estimates of solutions to elliptic equations in $\mathbf{R}^{n}$, in the half-space and in the unbounded dihedral angle. A detailed proof can be found in [14], [15].

Let $\sigma \in C^{\infty}(\mathbf{R})$ is such that $\sigma(t)=0$ for $|t|>1, \quad \sigma(t) \equiv 1$ for $|t|<\frac{1}{2}$.

Let $u(x) \in C^{2 m}(\bar{\Omega})$ be a function such that

$$
B_{j} u=0, \quad \text { on } \partial \Omega \backslash O, \quad j=1, \ldots m .
$$

Set

$$
v_{\mu}\left(x, x_{n+1}\right)=\sigma\left(x_{n+1}\right) e^{i \mu x_{n+1}} u(x),
$$

for any real $\mu$.

Denote $\Omega_{\rho}=\Omega_{0} \cap\left\{\left(x, x_{n+1}\right):\left|x_{n+1}\right|<\rho\right\}$. Using the inequality (5) we see that

$$
\begin{gathered}
\sum_{|\alpha| \leq 2 m_{\Omega_{1}}} \int_{r^{\gamma-4 m+2|\alpha|}\left|D^{\alpha} v_{\mu}\right|^{2} d x d x_{n+1}} \\
\leq C\left[\int_{\Omega_{1}}\left|\mathcal{L} v_{\mu}\right|^{2} r^{\gamma} d x d x_{n+1}+C \int_{\Omega_{1}}\left|v_{\mu}\right|^{2} d x d x_{n+1}\right] .
\end{gathered}
$$

Note that

$$
\mathcal{L} v_{\mu}=\sigma\left(x_{n+1}\right) e^{i \mu x_{n+1}}\left(L u-\mu^{2 m} e^{i \theta} u\right)+S\left(u e^{i \mu x_{n+1}}\right)
$$


where $S$ is a linear differential operator of order $(2 m-1)$ with bounded coefficients. Since $v_{\mu} \equiv 0$ for $\left|x_{n+1}\right|>1$, (6) implies that

$$
\begin{aligned}
\int_{\Omega_{1 / 2}} & \sum_{|\alpha| \leq 2 m} r^{\gamma-4 m+2|\alpha|}\left|D^{\alpha}\left(u e^{i \mu x_{n+1}}\right)\right|^{2} d x d x_{n+1} \\
\leq & C_{1}\left[\int_{\Omega}\left|L u-\mu^{2 m} e^{i \theta} u\right|^{2} r^{\gamma} d x_{i} \ldots d x_{n}\right. \\
& \left.+\sum_{|\alpha| \leq 2 m-1} \int_{\Omega} r^{\gamma} \mu^{2(2 m-1)}|u|^{2} d x_{1} \ldots d x_{n}+\int_{\Omega}|u|^{2} d x_{1} \ldots d x_{n}\right],
\end{aligned}
$$

where $C_{1}=$ const does not depend on $\mu$ and on $u$.

Besides,

$$
\begin{aligned}
& \sum_{|\alpha| \leq 2 m} C_{2} \int_{\Omega_{1 / 2}} r^{\gamma-4 m+2|\alpha|}\left|D^{\alpha} u e^{i \mu x_{n+1}}\right|^{2} d x_{1} \ldots d x_{n+1} \\
& \quad \geq \int_{\Omega} \sum_{|\alpha|=2 m} \sum_{s+|\beta|=|\alpha|} r^{\gamma-4 m+2|\alpha|}|\mu|^{2 s}\left|\frac{\partial^{\beta} u}{\partial x_{1}^{\beta_{1}} \ldots \partial x_{n}^{\beta_{n}}}\right|^{2} d x_{1} \ldots d x_{n} \\
& \quad \geq C_{3} \int_{\Omega} \sum_{|\beta| \leq 2 m} r^{\gamma}|\mu|^{2(2 m-|\beta|)}\left|\frac{\partial^{\beta} u}{\partial x_{1}^{\beta_{1}} \ldots \partial x_{n}^{\beta_{n}}}\right|^{2} d x_{1} \ldots d x_{n}+C_{3} \int_{\Omega}|\mu|^{-\gamma+4 m}|u|^{2} d x
\end{aligned}
$$

Therefore, if $|\mu|$ is large enough, we have

$$
\int_{\Omega}|u|^{2}|\mu|^{4 m-\gamma} d x \leq C_{4} \int_{\Omega}\left|L u-\mu^{2 m} e^{i \theta} u\right|^{2} d x,
$$

i.e.,

$$
|\lambda|^{\frac{4 m-\gamma}{2 m}}|| u\left\|_{L_{2}(\Omega)}^{2} \leq C_{5}|| L u-\lambda u\right\|_{L_{2}(\Omega)}^{2},
$$

if $\arg \lambda=\theta$ and $|\lambda|$ is large. This means that there are no points of the spectrum of the operator $L-\lambda E$ on the ray $\arg \lambda=\theta$ for sufficiently large $|\lambda|$ and

$$
\|\left.(\mathcal{L}-\lambda E)^{-1}\right|_{L_{2}(\Omega) \rightarrow L_{2}(\Omega)} \leq C|\lambda|^{-1+\frac{\gamma}{2 m}} .
$$

Since $0 \leq \gamma<2 m$ the ray arg $\lambda=\theta$ is a ray of minimal growth for the resolvent.

In order to finish the proof of Theorem 2 it remains to show that the map $\mathcal{L}-\lambda E$ is a map on the whole space $L_{2}(\Omega)$. This proof is long but it only employs standard methods of the theory of elliptic boundary problems with the help of a partition of the unity and the construction of a parametrix. 


\section{The growth of the resolvent}

Theorem 2 says that the spectrum of the operator $\mathcal{L}$ is discrete. Let us fix a point $z$ outside the spectrum of the operator $\mathcal{L}$, and set $T=(\mathcal{L}-z E)^{-1}$. We have

$$
R\left(\frac{1}{\lambda-z}, T\right)=(\lambda-z) E-(\lambda-z)^{2} R(\lambda, \mathcal{L}) .
$$

An element $\Phi \in L_{2}(\Omega), \Phi \neq 0$ is a generalized eigenelement of the operator $T$, corresponding to an eigenvalue $\mu$, if $(T-\mu)^{j} \Phi=0$ for some integer $j \geq 0$. The minimal $j$, for which this relation holds is called index of $\Phi$.

It is well-known that the dimension of the space of generalized eigenelements corresponding to an eigenvalue $\mu$ is finite. This dimension is called multiplicity of $\mu$. Let us denote $\mathcal{N}(T)$ the closure in $L_{2}(\Omega)$ of the linear envelope of all generalized eigenelements of the operator $T$.

The operator-valued function $R(\lambda, T)$ is a meromorphic function of $\frac{1}{\lambda}$ with its poles at the points which are eigenvalues of the operator $T$. Let $f \in L_{2}(\Omega)$. Let us consider the vector-valued function $R(\lambda, T) f$, which is analytic everywhere except for the point $\lambda=0$ and the points $\mu_{k}$, which can be its poles. If $\lambda=\mu_{k}$ is a pole of $R(\lambda, T) f$, then in a sufficiently small neighborhood of $\mu_{k}$ the Laurent expansion holds:

$$
R(\lambda, T) f=\frac{\Phi_{1}}{\left(\lambda-\mu_{k}\right)^{j}}+\frac{\Phi_{2}}{\left(\lambda-\mu_{k}\right)^{j-1}}+\cdots+\frac{\Phi_{j}}{\lambda-\mu_{k}}+\sum_{i=0}^{\infty} g_{i}\left(\lambda-\mu_{k}\right)^{i},
$$

where $j>1, \Phi_{1} \neq 0, \Phi_{j} \in L_{2}(\Omega), g_{j} \in L_{2}(\Omega), \Phi_{i}$ are generalized eigenelements $T$ of index $i$.

Similarly, the function $\Phi(x) \in D_{\mathcal{L}}$ is a generalized eigenelement of $\mathcal{L}$, corresponding to an eigenvalue $\lambda_{k}$, if $\left(\mathcal{L}-\lambda_{k}\right)^{j} \Phi=0$ for some $j \geq 1$. The minimal $j$, for which $\left(\mathcal{L}-\lambda_{k}\right)^{j} \Phi=0$ is also called index $\Phi$. It is clear that the function $\Phi$ is a generalized eigenelement of $\mathcal{L}$, corresponding to $\lambda_{k}$ if and only if $\Phi$ is a generalized eigenelement of $T$, corresponding to the eigenvalue $\frac{1}{\lambda_{k}-z_{0}}$. The closure in $L_{2}(\Omega)$ of the linear envelope of all generalized eigenelements of the operator $\mathcal{L}$ is denoted by $\mathcal{M}(\mathcal{L})$. We will show that $\mathcal{M}(\mathcal{L})=L_{2}(\Omega)$. First we show the following result about the growth of the resolvent, cf. Section 7 .

Theorem 3. Let $T$ be a compact operator in $L_{2}(\Omega)$ such that

$$
T L_{2}(\Omega) \subset W_{\gamma}^{2 m}(\Omega), \quad 2 m>\gamma>0 .
$$

Let $\lambda_{j}$ be a sequence of nonzero eigenvalues of $T$, counted with their multiplicity and $R(\lambda, T)$ be the resolvent of $T$. Then

1) $\sum_{j}\left|\lambda_{j}\right|^{\frac{n}{2 m-\frac{\gamma}{2}}+\varepsilon}<\infty$ for any $\varepsilon>0$. 
2) There exists a sequence $\rho_{i} \rightarrow 0, i=1,2 \ldots$ such that $R(\lambda, T)$ is defined for $|\lambda|=\rho_{i}$ and for any $\varepsilon>0$

$$
\|R(\lambda, T)\| \leq \exp \left(|\lambda|^{-\frac{n}{2 m-\frac{\gamma}{2}}+\varepsilon}\right), \text { for }|\lambda|=\rho_{i}, \quad i=1,2 \ldots
$$

Before we give the proof of Theorem 3 we shall state some constructions from [8].

Let $Q$ be a cube in $\mathbf{R}^{n}$,

$$
Q=\left\{x \in \mathbf{R}^{n}:\left|x_{i}\right|<\pi, i=1, \ldots, n\right\} .
$$

If $u \in L_{2}(Q)$ then

$$
u(x)=\sum_{k_{1}, \ldots, k_{n}} a_{k_{1}, \ldots, k_{n}} e^{i\left(k_{1} x_{1}+\cdots+k_{n} x_{n}\right)} .
$$

Let $H_{r}$ be the space of functions $u$ with a finite norm

$$
\|u\|_{r}^{2}=\left|a_{0}\right|^{2}+\sum_{k \neq 0}|k|^{2 r}\left|a_{k}\right|^{2}, \quad r>0
$$

Set

$$
\Lambda_{s} u(x)=\sum_{k_{1}, \ldots, k_{n}}\left(1+k_{1}^{2}+\cdots+k_{n}^{2}\right)^{s / 2} a_{k_{1}, \ldots, k_{n}} e^{i k_{1} x_{1}+\cdots+i k_{n} x_{n}} .
$$

It is easy to see that the operator $\Lambda_{-s}$ for $s>0$ is self-adjoint compact in $L_{2}(Q)$. Its eigenfunctions are $e^{i k_{1} x_{1}+\cdots+i k_{n} x_{n}}$ and the eigenvalues $\left(1+k_{1}^{2}+\cdots+k_{n}^{2}\right)^{s / 2}$.

Let $z_{0}$ be a point not belonging to the spectrum of $\mathcal{A}$. Put $T=\left(\mathcal{A}-z_{0} E\right)^{-1}$. We can assume that $z_{0}=0$. Put $R(\lambda, T)=(T-\lambda E)^{-1}$.

The operator $T^{*} T$ is nonnegative self-adjoint compact in $\mathcal{H}$. The operator $S=\left(T^{*} T\right)^{1 / 2}$ is also nonnegative self-adjoint compact in $\mathcal{H}$. Let $\mu_{i}(T)$ be the eigenvalues of the operator $S$.

Definition. The operator $T$ belongs to the class $C_{p}, 0<p<\infty$, if

$$
\sum_{i}\left|\mu_{i}(T)\right|^{p}<\infty
$$

Since

$$
\sum_{k_{1}, \ldots, k_{n}}\left(1+k_{1}^{2}+\cdots+k_{n}^{2}\right)^{-s p / 2}<\infty
$$

for $p>n / s$, the operator $\Lambda_{s}$ belongs to $C_{p}$ if $p s>n$.

The following Lemmas are taken from [8].

Lemma 1. Let $T$ be a compact linear operator $T: \mathcal{H} \rightarrow \mathcal{H}$ from the class $C_{p}$, $0<p<\infty$. Then there exists a sequence $\rho_{i}, \rho_{i} \rightarrow 0$ such that

$$
\|R(\lambda, T)\| \leq C \exp \left(c|\lambda|^{-p}\right)
$$


for $|\lambda|=\rho_{i}$.

Lemma 2. Let $T$ be a compact linear operator $T: \mathcal{H} \rightarrow \mathcal{H}$ from the class $C_{p}, 0<p<\infty$ and a linear operator $B$ be bounded as an operator from $\mathcal{H}$ to $\mathcal{H}$. Then the operators $B T$ and $T B$ belong to $C_{p}$.

Let us also recall the following important theorem:

Theorem 4. (see [8]) Let $T$ be a compact operator in the Hilbert space $H_{k}$ for some $k \geq 0$ and $T H_{k} \subset H_{k+s}$ for some number $s>0$. Then we have $T \in C_{n / s+\varepsilon}$ for any $\varepsilon>0$ and

$$
\|C(\lambda) R(\lambda, T)\| \leq \exp \left(c|\lambda|^{-n / s-\varepsilon}\right)
$$

for every $\varepsilon>0$ and $|\lambda| \leq \delta_{0}, \delta_{0}=\delta_{0}(\varepsilon)>0$, where

$$
C(\lambda)=\prod_{j}\left(1-\frac{\lambda_{j}}{\lambda}\right) \exp \left(\frac{\lambda_{j}}{\lambda}+\cdots+\frac{1}{l}\left(\frac{\lambda_{j}}{\lambda}\right)^{l}\right),
$$

and $l$ is the largest integer $\leq n / s$. The function $C(\lambda)$ is an entire function of $1 / \lambda$ vanishing at the points $\lambda_{j}$ only.

The following theorem follows readily from Theorem 4 .

Theorem 5. (see [13]) Let $T$ be a compact operator in a Hilbert space $H_{s}$ belonging to the class $C_{p}, 0<p<\infty$. Let $\lambda_{i}$ be the sequence of non-zero eigenvalues of $T$ counted with their multiplicity. Then there exists a sequence $\rho_{i}, \rho_{i} \rightarrow 0$ such that $R(\lambda, T)$ exists everywhere on $|\lambda|=\rho_{i}$ and

$$
\|R(\lambda, T)\| \leq \exp \left(c|\lambda|^{-p}\right)
$$

for $|\lambda|=\rho_{i}$.

We will show that Theorem 3 can be deduced from Theorem 5 .

\section{$6 \quad$ Proof of Theorem 3.}

Let us suppose that $\Omega$ is contained inside the cube $Q=\left\{x:\left|x_{i}\right|<\pi, i=1, \ldots n\right\}$. Denote $J_{\Omega}$ the operator of restriction of elements of $L_{2}(Q)$ on $L_{2}(\Omega)$. Let us show that there exists the operator of extension $P, u \rightarrow P u$, mapping $W_{\gamma}^{2 m}(\Omega)$ on $H^{2 m-\gamma / 2}(Q)$, where $H^{2 m-\gamma / 2}(Q)$ is the space of $2 \pi$ - periodic functions with the norm

$$
\|u\|_{H^{s}(Q)}^{2}=\left|a_{(0, \ldots 0)}\right|^{2}+\Sigma^{\prime}\left(k_{1}^{2}+\cdots+\left.k_{n}^{2}\right|^{s}\left|a_{k_{1}} \ldots a_{k_{n}}\right|^{2}\right.
$$


where

$$
u=\sum_{k_{1} \ldots k_{n}}^{\prime} a_{k_{1} \ldots k_{n}} e^{i\left(k_{1} x+\cdots+k_{n} x_{n}\right)}
$$

is the expansion of $u(x)$ in the Fourier series, $\Sigma^{\prime}$ means the sum over all $k=$ $\left(k_{1}, \ldots, k_{n}\right)$ except for $k=0 ; s=2 m-\gamma / 2$.

Let us consider in $K$ the following partitions of unity:

$$
1=\sum_{i=-\infty}^{+\infty} \theta_{i}(x), \quad 1=\sum_{i=-\infty}^{i+\infty} \sigma_{i}(x)
$$

where $\theta_{i}(x) \in C^{\infty}(K), \sigma_{i}(x) \in C^{\infty}(K)$, supp $\theta_{i} \subset K_{a 2^{-i-1}, a 2^{-i}}$, supp $\sigma_{i} \subset$ $K_{a 2^{-i-2}, a 2^{-i+2}}, K_{a, b}=\{x: x \in K, a<|x|<b\}, K_{a}=K_{0, a},\left|D^{\alpha} \theta_{i}\right| \leq$ $C 2^{i|\alpha|},\left|D^{\alpha} \sigma_{i}\right| \leq C 2^{i|\alpha|}, \sigma=1$ in $K_{a 2^{-i-1}, a 2^{-i+1}}$.

Let $u_{1}=\theta u, u_{2}=(1-\theta) u$, where $\theta \in C^{\infty}\left(\mathbf{R}^{n}\right), \theta=1$ in a neighborhood of the point $x=0, \theta=0$ for $|x| \geq a$. Set $S_{a, b}=\{x: a<|x|<b\}$ and let $\Pi_{0}$ be a linear bounded operator of extension from $W_{2}^{k}\left(K_{a / 4,4 a}\right)$ to $W_{2}^{k}\left(S_{a / 4,4 a}\right)$. The operator $\Pi_{0}$ induces an operator $\Pi_{j}$ of extension from $W_{2}^{k}\left(K_{a 2^{-j-2}, a 2^{-j+2}}\right)$ to $W_{2}^{k}\left(S_{a 2^{-j-2}, a 2^{-j+2}}\right)$.

Such an operator can be defined in the following way. Let $u^{\prime}(x)=u\left(x 2^{j}\right), x \in$ $K_{a / 4,4 a}, u^{\prime \prime}(x)=\Pi_{0} u^{\prime}(x), \Pi_{j}(x)=u^{\prime \prime}\left(x 2^{-j}\right), x \in K_{a 2^{-j-2}, a 2^{-j+2}}$.

It is easy to see that

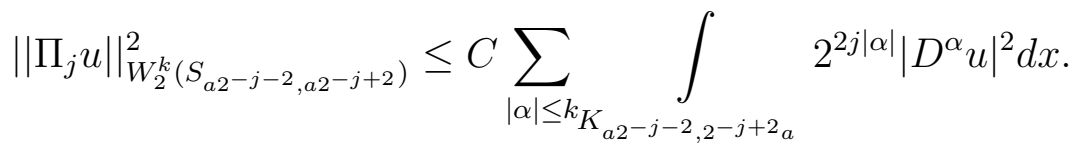

Now we can construct the extension $P u$ from $K$ to $\mathbf{R}^{n}$. Set

$$
P u=\sum_{i=0}^{+\infty} \sigma_{i} \Pi_{i} \theta_{i} u .
$$

The operator $P$ is an operator of extension from $K_{a / 2}$ to $S_{a / 2}$.

If $|\beta| \leq 2 m$, then we have obviously that

$$
\begin{aligned}
& \int_{S_{a / 2}}\left|D^{\beta} P u\right|^{2}|x|^{\gamma-2 m+2|\beta|} d x
\end{aligned}
$$

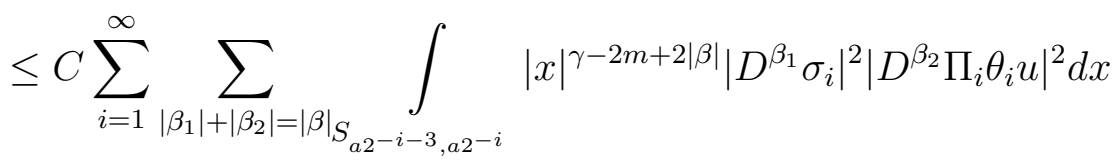

$$
\begin{aligned}
& \leq C_{1} \sum_{i=1}^{\infty} \sum_{\left|\beta_{1}\right|+\left|\beta_{2}\right|=|\beta|_{S_{a 2}-i-3, a 2^{-i}}} \int 2^{-(\gamma-2 m+2|\beta|) i} \cdot 2^{2\left|\beta_{1}\right| i}\left|D^{\beta_{2}} \Pi_{i} \theta_{i} u\right|^{2} d x
\end{aligned}
$$




$$
\begin{aligned}
& \leq C_{2} \sum_{i=1}^{\infty} \sum_{\left|\beta_{1}\right|+\left|\beta_{2}\right|=|\beta|} \int_{K_{a 2^{-i-2, a 2^{-i-1}}}} 2^{-(\gamma-2 m+2|\beta|) i+2\left|\beta_{i}\right| i}\left|D^{\beta_{2}} \theta_{i} u\right|^{2} d x \\
& \leq C_{3} \sum_{i=1}^{\infty} \sum_{\left|\beta_{1}\right|+\left|\beta_{2}\right|=|\beta|} \sum_{\left|\beta_{3}\right|+\left|\beta_{4}\right|=\left|\beta_{2}\right|_{K_{a 2^{-i-2}, a 2^{-i-1}}}} \int^{-(\gamma-2 m+2|\beta|) i+2\left|\beta_{1}\right| i+2\left|\beta_{3}\right| i}\left|D^{\beta_{4}} u\right|^{2} d x \\
& \leq C_{4} \sum_{i=1}^{\infty} \sum_{\left|\beta_{1}\right|+\left|\beta_{2}\right|=|\beta|} \sum_{\left|\beta_{3}\right|+\left|\beta_{4}\right|=\left|\beta_{2}\right|} \int_{K_{a 2^{-i-2}, a 2^{-i-1}}}|x|^{\gamma-2 m+2|\beta|-2\left|\beta_{1}\right|-2\left|\beta_{3}\right|}\left|D^{\beta_{4}} u\right|^{2} d x \\
& \leq C_{5} \sum_{\left|\beta_{4}\right| \leq 2 m} \int_{K}|x|^{\gamma-2 m+2\left|\beta_{4}\right|}\left|D^{\beta_{4}} u\right|^{2} d x \leq C_{6}\|u\|_{W_{\gamma}^{2 m}(K)}^{2} \text {. }
\end{aligned}
$$

The function $\hat{u}$ which is equal to $u$ in $\Omega$ and $P u$ in $S_{a / 2}$, belongs to $W_{\gamma}^{2 m}(\Omega \cap$ $\left.S_{a / 2}\right)$. The domain $\Omega \cap S_{a / 2}$ is Lipschitz, and we can expand $\hat{u}$ in the cube $Q=$ $\left\{x:\left|x_{i}\right| \leq a, i=1, \ldots, n\right\}$ in such a way that the continuation $u^{*}$ vanishes in a neighborhood of $\partial Q$ and belongs to $W_{\gamma}^{2 m}(Q)$. Moreover,

$$
\left\|u^{*}\right\|_{W_{\gamma}^{2 m}(Q)} \leq C_{7}\|u\|_{W_{\gamma}^{2 m}(\Omega)} .
$$

Define $u^{*}=0$ outside of $Q$. Let us check that $u^{*} \in H^{s}\left(\mathbf{R}^{n}\right)$ with $s<2 m-\gamma / 2$ and

$$
\left\|u^{*}\right\|_{H^{s}\left(R^{n}\right)} \leq C_{8}\|u\|_{W_{\gamma}^{2 m}(\Omega)} .
$$

To do that let $1=\sum_{i=-\infty}^{\infty} \psi_{i}(x)$ be a partition of unity in $\mathbf{R}^{n} \backslash 0, \psi \in$ $C_{0}^{\infty}\left(\mathbf{R}^{n}\right),\left|D^{\alpha} \psi_{i}\right| \leq C_{9} 2^{|\alpha| i}, \operatorname{supp} \psi_{i} \in S_{2^{-i}, 2^{1-i}}$. Set $u_{i}=\psi_{i} u^{*}$.

The interpolation inequality implies that

$$
\varepsilon^{2 s}|| u_{i} \|_{H^{s}\left(R^{n}\right)}^{2} \leq C_{10} \varepsilon^{4 m} \sum_{|\beta|=2 m_{R^{n}}} \int_{R^{n}}\left|D^{\beta} u_{i}\right|^{2} d x+C \int_{R^{n}}\left|u_{i}\right|^{2} d x,
$$

or

$$
\begin{aligned}
& \varepsilon^{2 s-4 m+\gamma}\left\|u_{i}\right\|_{H^{s}\left(R^{n}\right)}^{2} \\
& \quad \leq C_{11} \varepsilon^{\gamma} \sum_{|\beta|=2 m_{S_{2}-i, 2}} \int_{D^{-i+1}}\left|D^{\beta} u_{i}\right|^{2} d x+C_{11} \varepsilon^{\gamma-4 m} \int_{S_{2^{-i}, 2^{-i+1}}}\left|u_{i}\right|^{2} d x .
\end{aligned}
$$

Let $\varepsilon=2^{-i}$. Then

$$
\begin{aligned}
& \|\left. u_{i}\right|_{H^{s}\left(\mathbf{R}^{n}\right)} ^{2}
\end{aligned}
$$

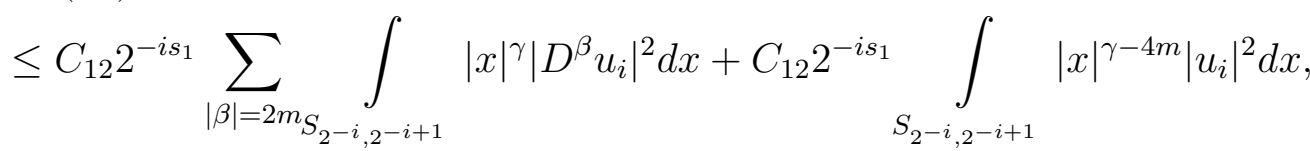

where $s_{1}=-\gamma+4 m-2 s$. 
Thus,

$$
\begin{aligned}
& \left\|u^{*}\right\|_{H^{s}\left(\mathbf{R}^{n}\right)} \leq \sum_{i=l}^{\infty}\left\|u_{i}\right\|_{H^{s}\left(\mathbf{R}^{n}\right)} \\
& \leq\left(\sum_{i=l}^{\infty} \sqrt{\sum\left\|u_{i}\right\|_{H^{s}\left(\mathbf{R}^{n}\right)}^{2} 2^{i s_{1}}}\right)^{\frac{1}{2}}\left(\sum_{i=1}^{\infty} 2^{-\frac{i s_{1}}{2}}\right)^{\frac{1}{2}} \\
& \leq C_{13}\left[\sum_{i=-l}^{\infty} \sum_{|\beta|=2 m_{S_{2^{-i}, 2^{-i+1}}}} \int_{S^{\gamma}}|x|^{\gamma}\left|u_{i}\right|^{2} d x+\int_{2^{-i}, 2^{-i+1}}|x|^{\gamma-2 m}\left|u_{i}\right|^{2} d x\right]^{\frac{1}{2}}
\end{aligned}
$$

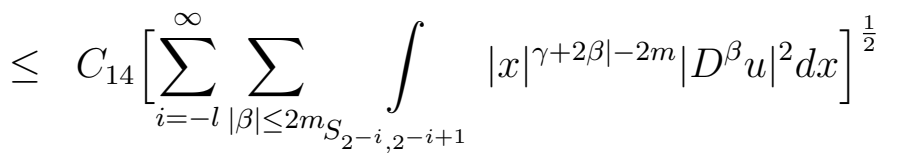

$$
\begin{aligned}
& \leq C_{15}\|u\|_{W_{\gamma}^{2 m}\left(\mathbf{R}^{n}\right)} \leq C_{16}\|u\|_{W_{\gamma}^{2 m}(\Omega)} \text {. }
\end{aligned}
$$

Therefore, the operator $P$ extends functions from $W_{\gamma}^{2 m}(\Omega)$ to $H^{s}\left(\mathbf{R}^{n}\right), s<$ $2 m-\gamma / 2$.

A function $u$ from $W_{\gamma}^{2 m}(Q) \cap H^{s}(Q)$, vanishing in a neighborhood of $\partial Q$ can be extended to $\mathbf{R}^{n}$ as a $2 \pi$ - periodic function. The norm of $u(x)$ in $H^{s}\left(\mathbf{R}^{n}\right)$ is equivalent to the norm (9), if we set $u(x)=0$ outside $Q$.

Let $T$ be a bounded linear operator $W_{\gamma}^{0}(\Omega) \rightarrow W_{\gamma}^{2 m}(\Omega)$.

Using the operator $P$ constructed before we can define the operator $T^{+}$in $L_{2}(Q)$ as

$$
T^{+} u=P T J u
$$

where $J$ is the operator of restriction of $L_{2}(Q)$ to $L_{2}(\Omega)$.

It is evident that the operator $T^{+}$is a compact operator from $L_{2}(Q)$ to $H_{s}(Q)$. Let us show that a $\lambda \neq 0$ belongs to the spectrum of the operator $T$ if and only if it belongs to the spectrum of the operator $T^{+}$. Moreover, the multiplicity of $\lambda$ as the spectrum point of $T$ is the same as that of $T^{+}$.

First observe that if $u(x) \in W_{\gamma}^{2 m}(\Omega)$, then $T^{+} P u=P T u$. Therefore, for any polynomial $p(t)$ we have

$$
p\left(T^{+}\right) P u=P p(T) u .
$$

Moreover, if $u^{*} \in W_{\gamma}^{m}(Q)$, then

$$
p(T) J u^{*}=J p\left(T^{+}\right) u^{*} .
$$

Let $\lambda \neq 0$ be an eigenvalue of the operator $T$ and $\Phi(x) \in L_{2}(\Omega)$ be an associated function of order $k \geq 1$, i.e.

$$
(T-\lambda E)^{k} \Phi=0, \quad(T-\lambda E)^{k-1} \Phi \neq 0
$$

(if $k=1$ it is simply an eigenfunction). 
Consider the polynomial

$$
q_{k-1}(t, \lambda)=\sum_{j=0}^{k-1}(t-\lambda)^{k-1-j}(-\lambda)^{j} .
$$

It is clear that

$$
-(-\lambda)^{k} \Phi=\left[(T-\lambda E)^{k}-(-\lambda)^{k} E\right] \Phi=T q_{k-1}(T, \lambda) \Phi .
$$

Since $T$ is bounded as an operator from $L_{2}(\Omega)$ to $W_{\gamma}^{2 m}(\Omega)$, we see that $\Phi \in$ $W_{\gamma}^{2 m}(\Omega)$.

Let us show now that if a $\lambda \neq 0$ is a regular point of the operator $T$, then

$$
\begin{aligned}
\|R(\lambda, T)\|_{W_{\gamma}^{0}(\Omega) \rightarrow W_{\gamma}^{2 m}(\Omega)} & \leq C_{17}\left\|R\left(\lambda, T^{+}\right)\right\|_{W_{\gamma}^{0}(Q) \rightarrow W_{\gamma}^{2 m}(Q)} \\
& \leq C_{18}|\lambda|^{-1}\left(\|R(\lambda, T)\|_{W_{\gamma}^{0}(\Omega) \rightarrow W_{\gamma}^{2 m}(\Omega)}+1\right),
\end{aligned}
$$

where $C_{18}$ does not depend on $\lambda$.

It is easy to check that

$$
R(\lambda, T) J u^{*}=J R\left(\lambda, T^{+}\right) u^{*} \quad \text { for } \quad u^{*} \in W_{\gamma}^{0}(Q),
$$

where

$$
R(\lambda, T): W_{\gamma}^{0}(\Omega) \rightarrow W_{\gamma}^{2 m}(\Omega), \quad R\left(\lambda, T^{+}\right): W_{\gamma}^{0}(Q) \rightarrow W_{\gamma}^{2 m}(Q) .
$$

Indeed,

$$
\begin{aligned}
R(\lambda, T) J u^{*} & =R(\lambda, T) J\left(T^{+}-\lambda E\right) R\left(\lambda, T^{+}\right) u^{*} \\
& =R(\lambda, T)(T-\lambda E) J R\left(\lambda, T^{+}\right) u^{*} \\
& =J R\left(\lambda, T^{+}\right) u^{*}
\end{aligned}
$$

Let $u \in W_{\gamma}^{k}(\Omega)$ and $u^{*}=P u$ in (12). We obtain

$$
R(\lambda, T) u-=J R\left(\lambda, T^{+}\right)(P u) .
$$

This relation implies

$$
\|R(\lambda, T)\| \leq C_{19}\left\|R\left(\lambda, T^{+}\right)\right\|
$$

with a constant $C_{19}$ independent of $\lambda$.

Conversely, if $u^{*} \in W_{\gamma}^{0}(Q)$, then by the definition of the operator $T^{+}$and (10) we have

$$
\begin{aligned}
\lambda R\left(\lambda, T^{+}\right) u^{*} & =T^{+} R\left(\lambda, T^{+}\right) u^{*}-u^{*} \\
& =\operatorname{PTJR}\left(\lambda, T^{+}\right) u^{*}-u^{*} \\
& =\operatorname{PTR}(\lambda, T) J u^{*}-u^{*} .
\end{aligned}
$$


Therefore,

$$
\left\|R\left(\lambda, T^{+}\right)\right\|_{W_{\gamma}^{0}(Q) \rightarrow W_{\gamma}^{2 m}(Q)} \leq \frac{C_{20}}{|\lambda|}(1+\|R(\lambda, T)\|)_{W_{\gamma}^{0}(\Omega) \rightarrow W_{\gamma}^{0}(\Omega)},
$$

where $C_{20}$ is independent of $\lambda$. So inequalities (11) are proved.

The operator $T=P R(\lambda, \mathcal{L}) J_{Q}: H^{t}(Q) \rightarrow H^{t+2 m-\gamma / 2-\varepsilon}(Q)$ is continuous. Its spectrum coincides with the spectrum of the operator $R(\lambda, \mathcal{L})$. Moreover, the operator $R(\lambda, \mathcal{L})=J_{Q} T P$ satisfies the conditions of Theorems 4 and 5 with $s=2 m-\gamma / 2-\varepsilon$.

Theorem 3 follows now from Theorems 4 and 5 .

\section{The completeness of root functions}

Now we can state our main result: the theorem on the completeness of the system of the root functions of an elliptic boundary problem in a domain with a conical point on its boundary.

Theorem 6. Assume that there exist the rays $\arg \lambda=\theta_{i}, i=1, \ldots, N$ in the complex plane which satisfy the conditions of Theorem 2 , and the angles between the pairs of neighboring rays are $<\pi(2 m-\alpha / 2) / n$. Then the spectrum of the operator $\mathcal{L}$ is discrete, and the root functions form a complete system in $L_{2}(\Omega)$.

Proof. Theorem 2 implies that the spectrum of the operator $\mathcal{L}$ is discrete, and each the ray $\arg \lambda=\theta_{i}$ is a ray of minimal growth for the resolvent $R(\lambda, \mathcal{L})$ : $L_{2}(\Omega) \rightarrow L_{2}(\Omega)$. This means, in particular, that

$$
\|R(\lambda, \mathcal{L})\|_{L_{2}(\Omega) \rightarrow L_{2}(\Omega)}=O\left(|\lambda|^{-\delta}\right)
$$

as $|\lambda| \rightarrow \infty$ and $\delta>0$.

Suppose that there exists a function $f^{*} \in L_{2}(\Omega)$, orthogonal to all eigen and associated functions of the operator $\mathcal{L}$. We will show that $f^{*}=0$. This will imply that the system of the root functions is complete.

Suppose that the point $\lambda=0$ is regular for the operator $\mathcal{L}$. Consider the function

$$
F(\lambda)=\left(f^{*}, R\left(\frac{1}{\lambda}, T\right) f\right)
$$

where $T=\mathcal{L}^{-1}, f \in L_{2}(\Omega)$, with $(\cdot, \cdot)$ being the scalar product in $L_{2}(\Omega)$.

Since the resolvent of $\mathcal{L}$ is a meromorphic function with poles at the points of the spectrum of $\mathcal{L}$, the function $F$ is analytic near those $\lambda$ which are not eigenvalues of $\mathcal{L}$. We shall use the following relation between the resolvents of the operators $\mathcal{L}$ and $\mathcal{L}^{-\infty}$ :

$$
R\left(\frac{1}{\lambda}, \mathcal{L}^{-1}\right)=\lambda E-\lambda^{2} R(\lambda, \mathcal{L})
$$


Consider the expansion

$$
R(\lambda, T) f=\frac{\Phi_{1}}{\left(\lambda-\lambda_{k}\right)^{j}}+\frac{\Phi_{2}}{\left(\lambda-\lambda_{k}\right)^{j-1}}+\cdots+\frac{\Phi_{j}}{\lambda-\lambda_{k}}+\sum_{i=0}^{\infty} g_{i}\left(\lambda-\lambda_{k}\right)^{i}
$$

in a neighborhood of the point $\lambda=\lambda_{k}$, where $\lambda_{k}$ is a pole of $R$. Here $j \geq 1, \Phi_{1} \neq$ $0, \Phi_{i} \in L_{2}(\Omega), g_{i} \in L_{2}(\Omega), \Phi_{1}, \Phi_{2}, \ldots, \Phi_{j}$ is a chain of the associated functions. This expansion implies that $\lambda_{k}$ is a regular point of $F(\lambda)$, since $f^{*}$ is orthogonal to all $\Phi_{i}$. Therefore, $F(\lambda)$ is an entire function.

The relations (13), (14), (15) imply that

$$
|F(\lambda)| \leq C \exp \left(|\lambda|^{2-\delta}\right)
$$

for $|\lambda| \rightarrow \infty, \arg \lambda=\theta_{i}, i=1, \ldots, N$. Besides, Theorem 3 has the consequence that for any $\varepsilon>0$ there exists a sequence $r_{j} \rightarrow \infty$ such that

$$
|F(\lambda)| \leq \exp \left(|\lambda|^{n /(2 m-\gamma / 2)-\varepsilon}\right)
$$

for $|\lambda|=r_{j}$.

Consider $F(\lambda)$ in the closure of the angle between the rays $\arg \lambda=\theta_{j}$ and $\arg \lambda=\theta_{j+1}$. Its size is less than $\pi(2 m-\gamma / 2) / n$. Since

$$
R(\lambda, T)=\lambda E-\lambda^{2} R(\lambda, \mathcal{L})
$$

and the ray $\arg \lambda=\theta_{i}$ is a ray of minimal growth, we have the inequality (16) on the sides of the angle and (17) on the sequence of the arcs tending to infinity.

Choosing $\varepsilon>0$ in (17) sufficiently small and applying the Phragmen-Lindelöf theorem we obtain that $|F(\lambda)|=O\left(|\lambda|^{2-\delta}\right)$ as $|\lambda| \rightarrow \infty$ in the whole complex plane. Therefore, $F(\lambda)$ is a linear function, i.e.,

$$
F(\lambda)=c_{0}+c_{1} \lambda
$$

On the other hand, we have

$$
R(1 / \lambda, T)=\lambda E+\lambda^{2}+\ldots,
$$

and, therefore,

$$
F(\lambda)=\lambda\left(f^{*}, f\right)+\lambda^{2}\left(f^{*}, T f\right)+\ldots
$$

Since $F$ is linear, we have $\left(f^{*}, T f\right)=0$ for all $f \in L_{2}(\Omega)$. Since the range of the operator $\mathcal{L}$ is dense in $L_{2}(\Omega)$, it follows that $f^{*}=0$. Thus, the system of the root functions of the operator $\mathcal{L}$ is complete in $L_{2}(\Omega)$. 


\section{Some generalizations}

In the preceding section we have proved the completeness of the system of the eigen and associated functions in $L_{2}(\Omega)$. This theorem implies immediately the completeness in $W_{\gamma}^{0}(Q)$ with $\gamma>0$. Indeed, let $f \in W_{\gamma}^{0}(Q), \gamma>0$. Set $f_{\varepsilon}=0$ for $|x|<\rho, f_{\varepsilon}=f$ for $|x|>\rho$, where $\rho>0$ is so small that

$$
\left\|f-f_{\varepsilon}\right\|_{W_{\gamma}^{0}(\Omega)} \leq \varepsilon
$$

Since $f_{\varepsilon} \in L_{2}(\Omega)$ there exists a finite linear combination of the root vectors $P(x)$ such that

$$
\left\|f_{\varepsilon}-P(x)\right\|_{L_{2}(\Omega)} \leq \varepsilon / \rho^{\gamma} .
$$

Then

$$
\left\|f_{\varepsilon}-P(x)\right\|_{W_{\gamma}^{0}(\Omega)} \leq \varepsilon
$$

and

$$
\|f-P(x)\|_{W_{\gamma}^{0}(\Omega)} \leq 2 \varepsilon .
$$

Now we shall state some corollaries of Theorem 6 .

Corollary 1. Under the conditions of Theorem 6 the system of the root elements is dense in the space

$$
\tilde{W}_{\gamma}^{2 m}(\Omega)=\left\{u \in W_{\gamma}^{2 m}(\Omega), B u=0 \text { on } \partial \Omega\right\}
$$

for $\gamma \geq 0$.

Proof. Indeed, let $u \in \tilde{W}_{\gamma}^{2 m}(\Omega)$. Then $L u \in W_{\gamma}^{0}(\Omega)$. Therefore, for any $\varepsilon>0$ there exists a linear combination of the root elements $P(x)$ such that

$$
\|L u-P(x)\|_{W_{\gamma}^{0}(\Omega)} \leq \varepsilon .
$$

Let the point $\lambda=0$ be regular for the operator $\mathcal{L}$. We can assume this without loss generality. The function $P_{0}(x)=\mathcal{L}^{-1}(P(x))$ is also a linear combination of the root elements. It follows from (18) that

$$
\left\|u-P_{0}(x)\right\|_{W_{\gamma}^{2 m}(\Omega)} \leq C \varepsilon,
$$

where $C$ does not depend on $u$ and $\varepsilon$. The inequality (19) means that the system of the eigen and associated functions is dense in $W_{\gamma}^{2 m}(\Omega)$.

Corollary 2. Let the conditions of Theorem 6 be satisfied, $0 \leq \gamma_{1} \leq \gamma$, and assume that the strip

$$
(-\gamma+4 m-n) / 2 \leq \operatorname{Im} \lambda \leq\left(-\gamma_{1}+4 m-n\right) / 2
$$

does not contain points of the spectrum of the problem (2). Then the system of root elements is dense in the space $\tilde{W}_{\gamma_{1}}^{2 m}(\Omega)$. 
Proof. Indeed, let $u \in \tilde{W}_{\gamma_{1}}^{2 m}(\Omega)$. Then $L u \in W_{\gamma_{1}}^{0}(\Omega)$. Therefore, for any $\varepsilon>0$ there exists a linear combination of the root elements $P(x)$ such that

$$
\|L u-P(x)\|_{W_{\gamma_{1}}^{0}(\Omega)} \leq \varepsilon .
$$

Let the point $\lambda=0$ be regular for the operator $\mathcal{L}$ which can be assumed without loss of generality. The regularity theorem for solutions of an elliptic boundary value problem in domains with conical points on the boundary implies that the point $\lambda=0$ is regular for the operator $L: W_{\gamma_{1}}^{2 m}(\Omega) \rightarrow W_{\gamma_{1}}^{0}(\Omega)$.

The function $P_{0}(x)=\mathcal{L}^{-1}(P(x))$ is also a linear combination of the root elements. It follows from $(20)$ that

$$
\left\|u-P_{0}(x)\right\|_{W_{\gamma}^{2 m}(\Omega)} \leq C \varepsilon,
$$

where $C$ does not depend on $u$ and $\varepsilon$. Inequality (21) means that the system of the eigen and associated functions is dense in $W_{\gamma_{1}}^{2 m}(\Omega)$.

Remark. Since

$$
W_{\gamma_{1}-2 m}^{0}(\Omega) \subset W_{\gamma_{1}}^{2 m}(\Omega)
$$

and the space $C_{0}^{\infty}(\Omega)$ is dense in any $W_{\gamma_{1}-2 m}^{0}(\Omega)$ with $\gamma_{1} \geq 0$, we can conclude that the system of the root functions is dense in any space $W_{\gamma}^{0}(\Omega)$ with $\gamma \geq-2 m$.

The obtained results can be extended to the spaces $L_{p}(\Omega), p \geq 1$.

Indeed, let $1 \leq p<2$, assume the strip

$$
\frac{-\gamma_{1}+4 m-n}{2} \leq \operatorname{Im} \lambda \leq \frac{-\gamma+4 m-n}{2}
$$

does not contain the points of the spectrum of the problem (2) and that the conditions of Theorem 3 are fulfilled. Then the system of the root functions of the problem (2) is complete in $W_{\gamma_{1}, p}^{2 m}(\Omega)$ and in

$$
\widehat{W_{\gamma_{1}, p}^{2 m}}(\Omega), \quad p \leq 2,
$$

where $W_{\gamma_{1}, p}^{2 m}(\Omega)$ is defined with the help of the norm

$$
\|u\|_{W_{\gamma_{1}, p}^{2 m}(\Omega)}^{p}=\sum_{\mid \alpha \leq 2 m} \int_{\Omega} r^{2 m+\gamma_{1}-p|\alpha|}\left|D^{\alpha} u\right|^{p} d x,
$$

and $\widehat{W_{a_{1}, p}^{2 m}}(\Omega)$ is its subspace of functions, satisfying the boundary conditions

$$
\left.B_{j} u\right|_{\partial \Omega}=0, \quad j=1, \ldots m .
$$

This follows readily from the embedding $\widehat{W_{\gamma_{1}, p}^{2 m}}(\Omega) \subset \widehat{W_{\gamma_{1}}^{2 m}}(\Omega)$, the details of the proof are the same as in the article of S. Agmon [8].

Note that

$$
\widehat{W_{\gamma_{1}, p}^{2 m}}(\Omega) \subset W_{\gamma_{1}^{\prime}, p_{1}}^{0}(\Omega) \text {, if } n>4 m,
$$


where $p_{1}=\frac{2 n}{n-4 m}, \quad \gamma_{1}^{\prime}=\frac{\gamma p_{1}}{2 m}$. This is a consequence of the embedding theorem for the weight Sobolev space with the limit exponent. From (22) it follows the completeness of the root functions in $W_{\gamma_{1^{\prime}}, p_{1}}^{0}(\Omega)$, and therefore, in $\widehat{W_{\gamma_{1}+4 m, p_{1}}^{2 m}}(\Omega)$, where $p_{1}=\frac{2 n}{n-4 m}>2$. Iterating these arguments we can prove the completeness of the system of the root functions of the problem (2) in the spaces $L_{p}(\Omega), p \geq 1$.

Example. Consider an elliptic operator of second order :

$$
L u \equiv \sum_{i, j=1}^{n} a_{i j}(x) u_{x_{i} x_{j}}+\sum_{i=1}^{n} a_{i}(x) u_{x_{i}}+a_{0}(x) u
$$

where $a_{i j}(x), a_{i}(x), a_{0}(x)$ are continuous real functions, which is defined on the set of $C^{2}$-functions satisfying the homogeneous Dirichlet conditions in a domain with a finite number of conical points on its boundary. In the case, when there are no conical points and the coefficients are smooth the completeness was proved in [8]. In our case this follows from Theorem 6. Note that we cannot apply the methods using the quadratic form $(L u, u)_{L_{2}(\Omega)}$ as in [5], [6], since the coefficients $a_{i j}$ may be not differentiable.

\section{References}

[1] Browder F.E., On the eigenfunctions and eigenvalues of the general elliptic differential operator, Proc. Nat. Acad. Sci. USA, 39, 1953, pp. 433-439.

[2] Browder F.E., Estimates and existence theorems for elliptic boundary value problems, Proc. Nat. Acad. Sci. USA, 45, 1959, pp. 365-372.

[3] Browder F.E., On the spectral theory of strongly elliptic differential operators Proc. Nat. Acad. Sci. USA, 45, 1959, pp. 1423-1431.

[4] Keldysh M. V., On the eigenvalues and eigenfunctions of certain classes of non-selfadjoint equations, Doklady AN SSSR, 77, 1951, pp. 11-14

[5] Agranovich M. S., On series with respect to root vectors of operators associated with forms having symmetric principal part, Funct. Anal. Appl., 28, n.3 1994, pp. 151-167.

[6] Krukovsky N.M., Theorems on the $m$-fold completeness of the generalized eigen- and associated functions from $W_{2}^{1}$ of certain boundary value problems for elliptic equations and systems, Diff. Uravneniya, 12, n.10, 1976, pp. 18421851.

[7] Carleman T., Über die Verteilung der Eigenwerte partieller Differentialgleichungen, Ber. der Sächs. Akad. Wiss. Leipzig, Mat. Nat. Kl., 88, 1936, pp. 119-132. 
[8] Agmon S., On the eigenfunctions and on the eigenvalues of general elliptic boundary value problems, Comm. Pure Appl. Math., 15, 1962, pp. 119-147.

[9] Schechter M., Remarks on elliptic boundary value problems, Comm. Pure Appl. Math., 12, 1959, pp. 457-482.

[10] Agmon S., Douglis A., Nirenberg L., Estimates near the boundary for solutions of elliptic partial differential equations satisfying general boundary conditions, Comm. Pure Appl. Math., 12, 1959, pp. 623-727.

[11] Agranovich M. S., Denk R., Faierman M., Weakly smooth nonselfadjoint spectral problems for elliptic boundary value problems, in the book : Spectral Theory, Microlocal Analysis, Singular Manifolds, Edited by P. Demuth, E. Schrohe, B.-W. Schulze, Birkh auser, 2000, pp. 138-199.

[12] Agranovich M. S., Elliptic Boundary Problems, in the book : Partial Differential Equations IX, Encyclopedia of Mathematical Sciences, vol. 79, pp. 1-144, Springer, 1991.

[13] Dunford N., Schwartz J.T., Linear Operators, vol II,

[14] Kondratiev V.A., Trudy Mosk. Mat. Ob., Boundary value problems for elliptic equations in domains with conical or singular points, 16, 1967, pp. 209-292.

[15] Egorov Yu.V., Schulze B.-W., Pseudo-differential Operators, Singularities, Applications, Birkhäuser, Operator Theory, Advances and Applications, vol. 93, 1997

[16] Gil J.B., Full asymptotic expansion of the heat trace for non-self-adjoint elliptic cone operators, Math. Nachr., 250, 2003, pp. 25-57.

[17] Schulze B.-W., Pseudo-differential operators on manifolds with singularities, North-Holland, Amsterdam, 1991.

[18] Schulze B.-W., Boundary value problems and singular pseudo-differential operators, J. Wiley, Chichester, 1998.

[19] Maz'ya V. G., Plamenevskii B. A., On the asymptotics of the fundamental solutions of elliptic boundary value problems in domains with conic points, Probl. Math. Anal., 7, 1979, pp. 100-145.

[20] Nazarov S. A., Plamenevskii B. A., Elliptic Problems in Domains with Piecewise smooth Boundaries, De-Gruyter Expositions in Mathematics, 13, 1994, Berlin, New York. 
[21] Egorov Yu.V., Kondratiev V.A., Schulze B.-W., Completeness of eigenfunctions of an elliptic operator on a manifold with conic points, Russian Journal of Mathematical Physics, 8, n.3, 2001, pp. 267-274. 